\section{Inaugural Editorial}

\author{
James O'Higgins Norman ${ }^{1}$ - Sameer Hinduja ${ }^{2}$
}

Published online: 12 March 2019

(C) Springer Nature Switzerland AG 2019

\section{Introduction}

Over the last few years, something tremendously exciting has occurred at a global level in relation to the problem of bullying (in almost every sphere and setting of social interaction). It is apparent that identification, prevention, and response efforts in this field have expanded beyond a scattered assortment of networks and projects to begin to form what we can now recognise as a truly global community of scholars and practitioners working together to tackle bullying, cyberbullying, and related forms of peer aggression. Families, schools, workplaces, and communities across the world are greatly (and increasingly) impacted by these issues, and are constantly searching for best practices that can meaningfully help. Indeed, even the political heads of entire countries, major international NGOs, and for-profit corporations are demonstrating a deep interest in further understanding and addressing the problems at hand.

The formation of this global community of scholars and practitioners (many of whom are part of the International Bullying Prevention Association (ibpaworld.org)) and the World Anti-Bullying Forum (wabf2019.com) heralds the arrival of bullying studies as a distinct, robust field of inquiry no longer competing for space and attention within other established disciplines. It is in this context that we recognised the need for a new peer-reviewed scholarly journal focused entirely on bullying prevention. This new journal will provide a space in which scholars and practitioners alike will share new theoretical and empirical insights into the issues at hand.

James O’Higgins Norman

james.ohigginsnorman@dcu.ie

Sameer Hinduja

hinduja@fau.edu

1 National Anti-Bullying Research and Resource Centre, Dublin City University, Dublin, Ireland

2 Florida Atlantic University, Jupiter, FL 33458, USA

\section{Our Goals}

Given that there has been so much disparate research on bullying, it is essential that any new publications on this topic move the conversation forward and enhance not just our conceptual and methodological knowledge, but also our practical skills in this area. The papers that will be published in each quarterly issue will reflect research by academics and practitioners in a variety of settings. As such, the journal not only makes a very significant contribution to the established body of knowledge on bullying but will assist educators, parents, students, employees, managers, mental health professionals, paediatricians, policymakers, and even tech companies by contributing new strategies of addressing bullying to their toolbox.

\section{What We Publish}

We have conceptualised bullying prevention to include both preventing and intervening at any stage in the process of bullying, cyberbullying, and related forms of aggression. While not wishing to confine authors to just one definition of bullying, the journal does operate out of an understanding that bullying, face-to-face or online, always involves aggression, repetition, intentionality, and a power imbalance.

We encourage submissions that extend existing definitions of bullying and seek to explore new theories that will help us to understand bullying. It is critical that we understand bullying not just as a psychological problem but equally as a sociological, philosophical, educational, and technological problem-and break through existing silos in our quest for a fuller understanding of all possible contributive factors and consequences. Such a shift in how we understand bullying involves a recognition that while certain individuals are more likely to bully, the context in which they operate often contributes towards their attitudinal and behavioural choices. 
We also encourage submissions about evidence-based initiatives aimed at tackling bullying in a variety of contexts including, but not limited to, schools, workplaces, universities, youth organisations, and of course in cyberspace. Given our increased awareness of the complex and multifaceted impact of bullying on individuals and communities, it is our expectation that the research and initiatives featured in this journal will serve as a valuable resource for the iterative development of future anti-bullying and online safety programmes and policy for practitioners.

\section{Our Inaugural Issue}

The papers included in this inaugural issue of the International Journal of Bullying Prevention have been chosen to reflect what we see as important for the future of the journal and the global community of scholars who will sustain the scholarship contained in the journal. These papers are representative of different approaches, cultures, and contexts and together they hail a new era in scholarship about bullying, cyberbullying, and peer aggression.

In the first paper by Dr. Peter Smith and Susanne Robinson MSc, the authors build on previous theoretical work done on the relationship between aggression and bullying to societies characterised by individualism or collectivism. Their findings regarding the role of regulatory frameworks and resources are important for policymakers in different countries.

The second paper by Hannah Gaffney MSc, Dr. David P. Farrington, and Dr. Maria M. Ttofi presents results from an extensive systematic and metaanalytical review of the effectiveness of school-based bullying prevention programmes in 12 countries. Their insights into the effectiveness of these interventions show that the effectiveness of school-based interventions for bullying perpetration and victimisation varies depending on location. Understanding this can help educators create and implement more context-specific solutions on the front lines.

The third paper in this inaugural issue by Yuanhong Huang MA, Dr. Dorothy L. Espelage, Dr. Joshua R. Polanin, and Dr. Jun Sung Hong provides a metaanalysis of prevention programmes that include a parental component. Their results show a modest but significant positive difference when parents are involved, highlighting the need for those who design prevention programmes to include a meaningful focus on equipping and empowering the family to share the load when reaching and teaching youth.

The fourth paper by Dr. Donna Cross, Dr. Kevin C. Runions, Dr. Therese Shaw, Dr. Janice WY Wong, Dr.
Marilyn Campbell, Dr. Natasha Pearce, Dr. Sharyn Burns, Dr. Leanne Lester, Dr. Amy Barnes, and Dr. Ken Resnicow reports on the implementation of the Friendly Schools intervention in secondary-level schools. Their study showed a significant decrease in reported bullying and a significant reduction in bullying victimisation and cybervictimisation when the studentcentric curriculum was delivered by endogenous providers (e.g., school staff and the educational publisher) within naturalistic conditions.

In the fifth paper by Dr. Elise Pas, Dr. Tracy E. Waasdrop, and Dr. Catherine Bradshaw, a mixed-reality simulation setup to allow teachers to practice identifying, preventing, and responding to classroom bullying was examined. This effort was informed by the knowledge that educators who receive regular and specific coaching supports are more successful in their classroom management goals (in this case, addressing student-based aggression).

Finally, in the sixth paper in this inaugural issue, Dr. Susan Limber, Dr. Dan Olweus, and Dr. Kyrre Breivik sought to evaluate the effectiveness of the Olweus Bullying Prevention Programme (OBPP) in reducing verbal bullying, physical bullying, and indirect/relational bullying, as well as cyberbullying and bullying using words or gestures with a sexual meaning. The findings of this longitudinal study involving over 30,000 students in grades 3-11 indicate that the programme is broadly useful, with stronger effects shown in schools where implementation has occurred over a longer period of time.

\section{Our Ultimate Intent}

It is our hope that you will not only find the papers in this inaugural issue of this journal to be interesting and stimulating, but that you will be inspired to undertake and submit your own research and evaluation to this journal. We are confident that each piece we publish will contribute in some notable way - not only to our understanding of these problem behaviours, but to our successful engagement with them. This will be the legacy of our efforts, and this will serve as our collective role towards fostering and maintaining a more civil society for all-irrespective of where we live, work, learn, or connect.

Please see our General Call for Papers (https://www. springer.com/psychology/child+\%26+school+psychology/ journal/42380) and join the journal's official Association-the International Bullying Prevention Association (https:// ibpaworld.org/). Finally, follow us on social media to stay in the loop about Special Issue Calls for Papers (https://www. springer.com/psychology/child+\&+school+psychology/ journal/42380?detailsPage $=$ societies) and other pertinent updates. 